\title{
A cost effectiveness analysis of the preferred antidotes for acute paracetamol poisoning patients in Sri Lanka
}

\author{
S M D K Ganga Senarathna ${ }^{1,2,3^{*}}$, Shalini Sri Ranganathan ${ }^{1}$, Nick Buckley ${ }^{2,4}$ and Rohini Fernandopulle
}

\begin{abstract}
Background: Acute paracetamol poisoning is a rapidly increasing problem in Sri Lanka. The antidotes are expensive and yet no health economic evaluation has been done on the therapy for acute paracetamol poisoning in the developing world. The aim of this study is to determine the cost effectiveness of using $\mathrm{N}$-acetylcysteine over methionine in the management of acute paracetamol poisoning in Sri Lanka.

Methods: Economic analysis was applied using public healthcare system payer perspective. Costs were obtained from a series of patients admitted to the National Hospital of Sri Lanka with a history of acute paracetamol overdose. Evidence on effectiveness was obtained from a systematic review of the literature. Death due to hepatotoxicity was used as the primary outcome of interest. Analysis and development of decision tree models was done using Tree Age Pro 2008.

Results: An affordable treatment threshold of Sri Lankan rupees 1,537,120/death prevented was set from the expected years of productive life gained and the average contribution to GDP. A cost-minimisation analysis was appropriate for patients presenting within 10 hours and methionine was the least costly antidote. For patients presenting 10-24 hours after poisoning, n-acetylcysteine was more effective and the incremental cost effectiveness ratio of Sri Lankan rupees 316,182/life saved was well under the threshold. One-way and multi-way sensitivity analysis also supported methionine for patients treated within 10 hours and n-acetylcysteine for patients treated within 10-24 hours as preferred antidotes.
\end{abstract}

Conclusions: Post ingestion time is an important determinant of preferred antidotal therapy for acute paracetamol poisoning patients in Sri Lanka. Using n-acetylcysteine in all patients is not cost effective. On economic grounds, methionine should become the preferred antidote for Sri Lankan patients treated within 10 hours of the acute ingestion and n-acetylcysteine should continue to be given to patients treated within 10-24 hours.

\section{Background}

Paracetamol is the most common cause of drug poisoning in the world [1] and the single most commonly taken drug in overdoses that lead to hospital presentation and admission [2].

Poisoning with paracetamol is an emerging problem in Sri Lanka with rapidly increasing admissions to the National Hospital of Sri Lanka (NHSL): from only 35 cases in 2003 to 515 cases in 2005 [3]. Paracetamol poisoning is one of the most expensive poisonings

\footnotetext{
* Correspondence: ganga136@yahoo.com

'Department of Pharmacology, Faculty of Medicine, University of Colombo,

Colombo, Sri Lanka

Full list of author information is available at the end of the article
}

management in Sri Lanka [4,5]. The average cost of managing a patient with acute paracetamol poisoning was even higher than the average cost of managing a organophosphate poisoning patient; the most costly poisoning management at the Anuradhapura General Hospital in Sri Lanka [4,5]. However it is important to note that eighty percent of the current total cost of management of acute paracetamol poisoning is due to cost of antidotes unlike in organophosphate poisoning [5].

Even though paracetamol poisoning has been researched far more than other pharmaceutical poisonings, there is limited literature on the pharmaco-economics of treatment. So far no full economic evaluation on interventions on paracetamol poisoning has been

\section{Biomed Central}


carried out [6]. Two antidotes, $\mathrm{N}$-acetylcysteine (NAC) and methionine, are available in Sri Lanka. NAC is the most expensive and also the most commonly used antidote. The evidence on effectiveness of antidotes used in acute paracetamol poisoning is weak [7]. However, on the basis of the current best available evidence NAC is considered to be more effective than methionine in the management of patients with acute paracetamol poisoning [7]. Considering that NAC is also more costly than providing methionine, it should be useful to determine the comparative cost effectiveness of the two treatments, and whether the additional benefit of NAC over methionine is worth the extra cost. Cost effectiveness analysis is based on the premise that it is the wider community interest which is paramount; therefore extra lives saved from use of a more expensive antidote have to be balanced against the extra costs involved in doing so. It is easy to make the management decision; if an intervention is dominant (i.e. the new intervention is less costly and yields higher benefit). But in situations where an intervention is not dominant, we have to find out the point at which the intervention is cost effective. The question of an intervention is cost-effective depends upon whether the relevant decision maker is willing and able to pay the additional costs to achieve the additional benefits that can be achieved by introducing the alternative program. The magnitude of this value will be controversial. In this analysis it was considered that the program is cost effective if it can gain a year of healthy life for less than a country's national income per person per capita gross national income [8].

The objective of the present cost effectiveness analysis is to determine the incremental cost effectiveness of NAC over methionine in the management of acute paracetamol poisoning patients (with suicidal intent) in Sri Lanka.

\section{Methods}

\section{Structure of the decision tree model}

The evidence on effectiveness of both antidotes; NAC and methionine depends on the post ingestion time. According to the best available evidence, all antidotes are much more effective if given within 10 hours of the acute ingestion [7].

Therefore we constructed two decision tree models: for patients treated within 10 hours, and for patients treated within 10 to 24 hours after acute ingestion of paracetamol (Figure 1). Both models compared use of methionine and NAC in patients where the risk was assessed using plasma paracetamol levels according to the Rumack-Matthew nomogram.

Acute paracetamol poisoning can result in fulminant hepatic failure which can lead to death. The most frequently applied definition for significant paracetamol

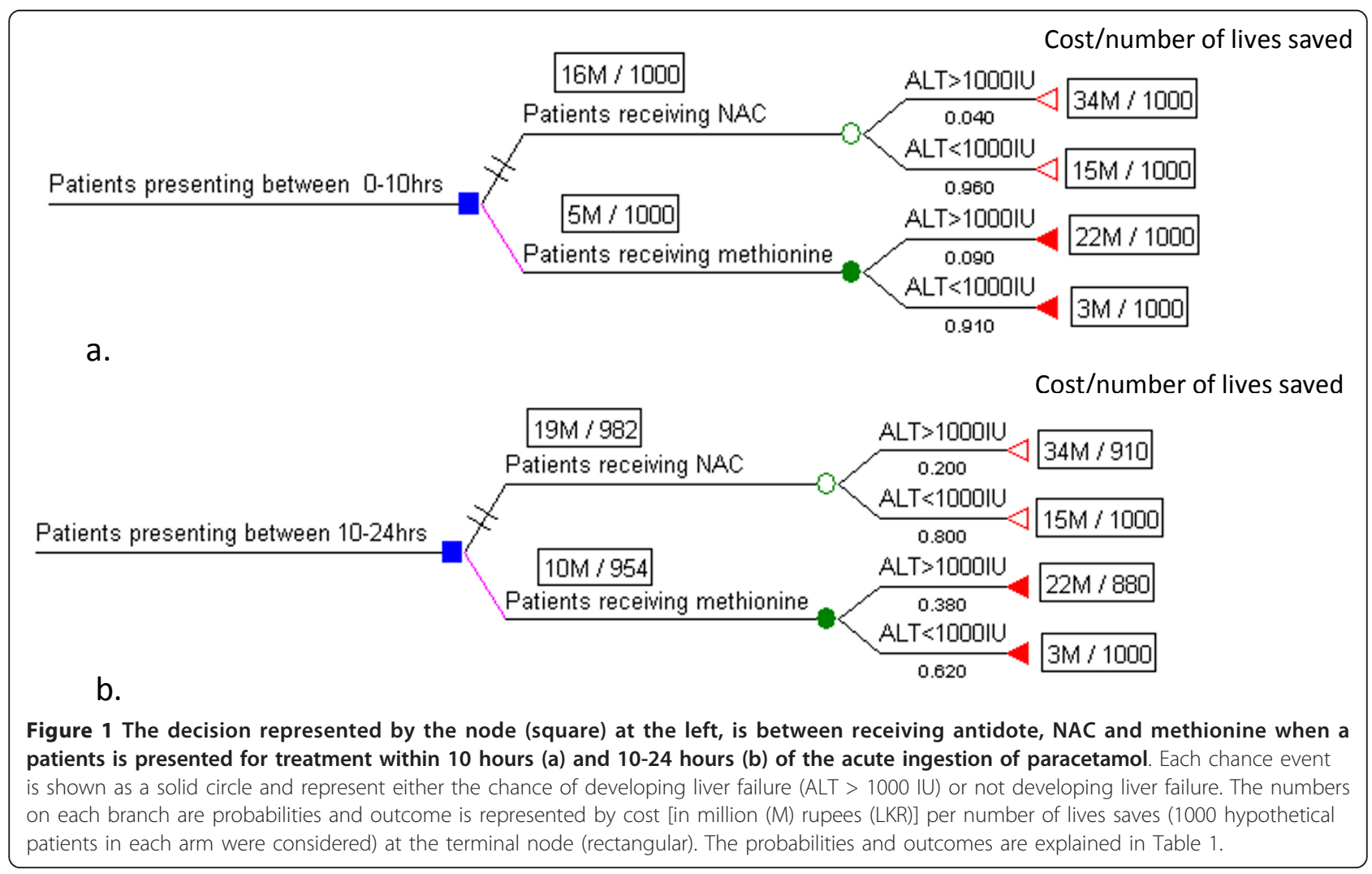


hepatotoxicity is having liver transaminases (ALT or AST) $>1000 \mathrm{U} / \mathrm{L}$ at any time $[9,10]$.

Therefore the decision tree model adopted death following hepatotoxicity (ALT or AST > $1000 \mathrm{U} / \mathrm{L}$ ) as the final outcome measure (Figure 1).

\section{Population considered}

The analysis was applied to patients admitted to the National Hospital of Sri Lanka (NHSL). The ethics approval was obtained from ethical review boards of the NHSL and the Faculty of Medicine, Colombo. In Sri Lanka healthcare is free at the point of delivery. Therefore the analysis was done from the public healthcare system payers' perspective. The time horizon was 2006 and the analysis was performed with reference to 1000 hypothetical patients in each arm.

\section{Data and assumptions on costs and effectiveness of antidotes \\ Costs}

The direct costs linked to the treatment options were measured as the total cost of drug treatment and hospitalisation. The total cost of drug treatment includes the cost of NAC/methionine (obtained from the medical supplies division of Sri Lanka), cost of hospital stay (obtained from accounts branch of the NHSL and follow up of case series) and cost of person hours (obtained from accounts branch of the NHSL and follow up of case series). The cost of antidotal therapy was based on the usual NAC (IV NAC $300 \mathrm{mg} / \mathrm{kg}$ over 20.25 hours) and methionine regimen( $2.5 \mathrm{~g}$ every four hours for four doses) used in Sri Lanka. The costs in LKR (Sri Lankan rupees) (100 LKR $=1$ US dollar) of items included in the analysis are given in Additional file 1: annexure1.

\section{Effectiveness data}

Antidotal effectiveness data was obtained from the systematic review and meta-analysis on the interventions for paracetamol poisoning by Brok et al. 2002 and 2006 [6,7]. The meta analysis included randomised controlled trials (RCTs), quasi-RCTs, RCTs with volunteers and observational studies. According to the review, no RCTs were of high quality. Further, no RCT compared NAC with methionine or no treatment for the relevant time frame (within24 hours of the acute ingestion) for this analysis. The review provided an exploratory analysis on antidotes and gave pooled probabilities for developing hepatotoxicity and death following acute paracetamol poisoning in patients with plasma paracetamol levels above possible or probable risk line of the nomogram $[10,11]$. Summary of the studies included in the exploratory analysis are given in Additional file 2: annexure 2 [9-21].

\section{Incremental cost effectiveness}

The total costs and outcomes for each treatment arm were presented. The expected value of each management alternatives identified at the root. The incremental cost effectiveness ratio (ICER) for two alternative treatments was calculated (incremental analysis produces a summary measure of relative efficiency) by dividing the cost difference by the outcome difference.

ICER $=($ Cost of NAC - cost of methionine $) /($ Outcome for NAC - outcome for methionine $)$

When there was no difference in outcome between management alternatives, cost minimisation analysis was done and the least costly management alternative was chosen.

Development of decision tree models and the analysis were done using Tree Age Pro Excel healthcare 2008 software, Serial number GXT2J-2B6KK-3D3DV-G.

\section{Discounting and sensitivity analysis}

Acute paracetamol poisoning is an acute condition and costs and outcomes occur during a short span of time: two to ten days. Therefore costs or outcomes were not discounted to adjust for elapsed time between expenditure and outcome when ICERs are calculated. When treatment threshold value was calculated, future income of the study patients were considered and therefore it was discounted at the rate of 3.5\% to bring it to the present value [22],

One-way-sensitivity analysis was done by increasing and decreasing costs and mortality by $50 \%$ and also by taking the upper and lower confidence interval of the probability on the systematic review estimates of mortality and hepatotoxicity with each antidote. Multi- ways sensitivity analysis was done to examine the worst combination of the single factors for both NAC and methionine in both time periods.

\section{Results}

Medical care cost

When a $60 \mathrm{~kg}$ patient is treated with NAC and does not develop hepatotoxicity, the cost to the healthcare system was 15,038 rupees. However if the patient develops hepatotoxicity, the cost rises to 34,329 rupees due to infusion of additional NAC and supportive care. The total cost for the methionine option was 2,839 rupees when patients do not develop hepatotoxicity and 22,481 when the patient develops hepatotoxicity. The breakdown on direct medical care costs are given in Additional file 1: annexure 1 .

\section{Outcome data}

The exploratory analysis of the systematic review pooled studies together and gave pooled probabilities as shown in Table 1[7]. The probability for death following the use of both antidotes was zero for patients treated within 10 hours of the acute ingestion. However, the $95 \% \mathrm{CI}$ of the case-fatality had an upper CI of $0.5 \%$ for NAC and $2.4 \%$ for methionine. 
Table 1 Probability for hepatotoxicity and death, when antidotes are administered within 0-10 and 10-24 hours from the acute ingestion

\begin{tabular}{|c|c|c|c|}
\hline & $\begin{array}{c}\text { Probability for } \\
\text { AST/ALT > } 1000 \text { IU/I (n/N) } \\
{[6,7]}\end{array}$ & Probability for death $(n / N)$ & Note \\
\hline \multicolumn{4}{|c|}{ IV NAC $300 \mathrm{mg} / \mathrm{kg}$ over 20.25 hours } \\
\hline \multirow[t]{2}{*}{ Within 10 hours } & $4 \%(13 / 315)$ & $0^{\dagger}(0 / 13)$ & * \\
\hline & (95\% Cl 2.5 to 7.0$)$ & (95\% Cl 0 to 24$)$ & \\
\hline \multirow[t]{2}{*}{ Within $10-24$ hours } & $20 \%(67 / 322)$ & $9 \%^{\ddagger}(6 / 67)$ & * \\
\hline & (95\% Cl 17 to 26$)$ & ( $95 \% \mathrm{Cl} 3.8$ to 18$)$ & \\
\hline \multicolumn{4}{|c|}{ Methionine $1 \mathrm{~g} 4$ hourly 4 doses } \\
\hline \multirow[t]{2}{*}{ Within 10 hours } & $9 \%(13 / 143)$ & $0^{+}$ & $\#$ \\
\hline & (95\% Cl 4.5 to 15$)$ & (95\% Cl 0 to 24$)$ & \\
\hline \multirow[t]{2}{*}{ Within $10-24$ hours } & $38 \%(17 / 41)$ & $12 \%^{\Pi}(2 / 17)$ & \# \\
\hline & (95\% Cl 26.3 to 57.9$)$ & (95\% Cl 2 to 36$)$ & \\
\hline
\end{tabular}

* Probabilities were derived by pooling results of Prescott et al. 1979, Smilkstein et al. 1988, Burkhart et al. 1995, Buckley et al. 1999, Parker et al. 1990, Smilkstein et al. 1991, Woo et al. 2000, Ayonrinde et al. 2005 and Kerr et al. 2005 [9-17]. \# Probabilities were derived by pooling results of Prescott et al. 1979, Crome et al. 1976, Hamlyn et al. 1981, Prescott et al. 1976 and Vale et al. 1981 [9,18-21]. + No patient treated (NAC/methionine) within 10 hours died due to hepatototoxicity, therefore the probability of death due to hepatotoxicity was zero

\section{Threshold value}

The mean per capita income for a Sri Lankan in 2006 was LKR 6,463/month [23]. The average age for the case series of patients was 20 years and the average number of working years for a Sri Lankan is 55 years which is the retirement age. When patients poisoned with paracetamol dies at the age of 20 , the society will lose average earning at the rate of percapita income per month up to the retirement age. Therefore the discounted present value of a life saved at the age of 20 would be LKR 1,537,120

(Discounted present value $\left.=\sum^{35-1} 77556_{n}(1-0.035)^{\mathrm{n}}\right)$ and this value was used as the treatment threshold value to prevent a death due to acute paracetamol poisoning in Sri Lanka. Therefore the study considered that it is cost effective to prevent an additional death by NAC at a cost of $\leq$ LKR $1,537,120$.

\section{Baseline results}

The decision tree models with expected outcomes for patients presenting within 10 and 10-24 hours is given in Figure 1.

The incremental cost effectiveness ratios reveal how much it would cost to prevent an extra death by shifting to the more costly antidote (NAC) from the cheaper alternative antidote (methionine). When treated within 10 hours of the acute ingestion, the incremental cost for treatment with NAC over methionine came to LKR $11,588,680$, but outcome resulted from alternative interventions were similar. Therefore base line ICER was not calculated for this group and cost minimisation analysis was done (Table 2).

Cost effectiveness analysis was done for patients treated within 10 to 24 hours, the ICER for this group of patients was LKR 316,182 per life saved (below the threshold value) where the incremental cost was LKR 8,726,620 and additional lives saved by NAC was 28 (Table 2).

\section{Sensitivity analysis}

\section{One way sensitivity analysis}

Incremental cost effectiveness ratios for one way sensitivity analysis, taking mortality as outcome measure are given in Table 2.

\section{Multi-way-sensitivity analysis}

According to the base-case analysis, methionine was the lease costly antidote for patients treated within 10 hours and NAC had an ICER below the threshold value for patients treated within 10 to 24 hours after ingestion. The robustness of this decision was further tested by assessing the worse case for methionine when given within 10 hours and the worse case for NAC when given 10-24 hours in a multi-way sensitivity analysis.

Worse case analysis for methionine within 10 hours gave an ICER of LKR 2,718,784 for NAC. Worse case analysis for NAC given within 10-24 hours showed dominance for methionine where methionine saved 42 more lives at a lower cost.

\section{Discussion}

The most important factor influencing total cost in the management of acute paracetamol poisoning in Sri Lanka is the antidotal therapy. This cost pattern would also be observed in other developing country settings where hospital stay and human resources are cheaper. Economic modeling should inform the most cost effective way of using these two antidotes which can reduce the total cost of management in developing country settings. 
Table 2 Incremental cost per life saved for baseline data and following one way sensitivity analysis (Number of lives saved as the final outcome measure)

\begin{tabular}{|c|c|c|}
\hline & \multicolumn{2}{|c|}{ Incremental cost per life saved(LKR/Life saved) } \\
\hline & $0-10 \mathrm{hrs}$ & $10-24$ hrs \\
\hline Baseline results & Not applicable ${ }^{\ddagger}$ & 316,182 \\
\hline \multicolumn{3}{|c|}{ One way sensitivity analysis results } \\
\hline \multicolumn{3}{|l|}{ Increasing by $50 \%$} \\
\hline NAC deaths & Not applicable & 469,173 \\
\hline NAC cost & Not applicable ${ }^{\ddagger}$ & 565,805 \\
\hline Methionine deaths & Not applicable $e^{\ddagger}$ & 173,147 \\
\hline Methionine cost & Not applicable ${ }^{\ddagger}$ & 265,107 \\
\hline \multicolumn{3}{|l|}{ Decreasing by $50 \%$} \\
\hline NAC deaths & Not applicable ${ }^{\ddagger}$ & 238,432 \\
\hline NAC cost & Not applicable $e^{\ddagger}$ & 55,312 \\
\hline Methionine deaths & Not applicable ${ }^{\ddagger}$ & $1,818,046$ \\
\hline Methionine cost & Not applicable ${ }^{\ddagger}$ & 366,486 \\
\hline \multicolumn{3}{|c|}{ Upper confidence interval(Cl) } \\
\hline NAC deaths & $\underline{\text { Dominance for methionine }}^{\dagger}$ & 909,023 \\
\hline NAC hepatotoxicity & Not applicable ${ }^{\ddagger}$ & 445,229 \\
\hline Methionine deaths & $5,365,130$ & 73,456 \\
\hline Methionine hepatotoxicity & Not applicable ${ }^{\ddagger}$ & 94,349 \\
\hline \multicolumn{3}{|c|}{ Lower confidence interval(Cl) } \\
\hline NAC deaths & Not applicable ${ }^{\ddagger}$ & 229,648 \\
\hline NAC hepatotoxicity & Not applicable ${ }^{\ddagger}$ & 268,907 \\
\hline Methionine deaths & Not applicable & $\underline{\text { Dominance for methionine }}^{+}$ \\
\hline Methionine hepatotoxicity & Not applicable & 836,480 \\
\hline
\end{tabular}

The most suitable economic analysis for patients treated within 10 hours of the acute ingestion is the cost minimization analysis, as the evidence suggests the probability of death is zero for patients treated with either antidote. With this premise, methionine is clearly the least costly alternative for this group of patients. One-way sensitivity analysis and multi-way sensitivity analysis calculated ICERs (on instances where there is an outcome difference) were above our pre-defined threshold for an acceptable cost per life saved by NAC. Therefore, methionine is the antidote of choice for patients treated within 10 hours.

The ICER for NAC for patients presenting within 1024 hours was LKR 316,182 for a death prevented. This is a value much lower than the treatment threshold value and suggests the use of NAC in preference to methionine is very cost-effective. One way sensitivity analysis in all instances had ICERs lower than the threshold value except when the lower confidence interval for death following methionine was considered where it showed dominance for methionine.

Worse case analysis for NAC also indicated dominance for methionine. Therefore the conclusion ranged from dominance for methionine to NAC being cost effective. However in most of the sensitivity analysis and in the base case analysis the ICER for NAC was much lower than the threshold value. Therefore use of NAC for patients presenting within 10-24 hours of the acute ingestion appears to be a cost effective option in Sri Lanka.

The effectiveness data for the decision tree was based on the systematic review and meta analysis by Brok et al. 2006. The meta-analysis referred to 10 studies to produce estimates of effectiveness in respect to these two antidotes; five studies for NAC, four studies for methionine and one study on both antidotes. According to the levels of evidence and grade of recommendation proposed by Cook et al. 1992 [24], studies used for methionine were in grade $B(1)$, grade $C(1)$ and grade $D$ (2), studies used for effectiveness of NAC were in grade $\mathrm{B}(1)$, Grade $\mathrm{D}(2)$ and grade $\mathrm{E}(2)$ and the study on both NAC and methionine was grade D quality.

The meta-analysis did not use any studies which provided grade A recommendations and eight out of 10 studies were in grade D or worse. An RCT directly comparing these two antidotes would provide much better 
effectiveness data. Such a trial might be worthwhile and should be strongly supported. The trial could not easily be blinded due to the different routes of administration. However, the outcome measures are largely objective and there is a low likelihood of bias.

\section{Conclusions}

Post ingestion time is an important determinant of preferred antidotal therapy for acute paracetamol poisoning patients in Sri Lanka. Using $\mathrm{N}$-acetylcysteine in all patients is not cost effective.

If policy makers are wishing to utilise economic evaluations to improve decision making, the findings from this study suggest that, within the first 10 hours, the use of methionine may be more cost-effective than NAC for paracetamol poisoning in Sri Lanka. This would potentially more than halve the total expenditure on this increasingly common poisoning. $\mathrm{N}$-acetylcysteine should continue to be given to patients treated within 10-24 hours.

\section{Limitations}

The study recommends the use of methionine for patients treated within 10 hours of the acute ingestion. There is some literature suggestive of methionine induce adverse effects such as nausea and vomiting. However our prospective case series didn't identify such incidences even though 55 patients received methionine compared 68 of IV NAC. Two patients received both antidotes owing to unavailability of IV NAC at the time of patient admission [3].

\section{Additional material}

Additional file 1: Annexure 1. Cost per patient (body weight: $60 \mathrm{~kg}$ ) for different treatment alternatives.

Additional file 2: Annexure 2. Summary of the studies included in the exploratory analysis of the systematic review [9-21].

\begin{abstract}
Abbreviations
GDP: Gross domestic product; NHSL: National Hospital of Sri Lanka; NAC: Nacetylcysteine; ALT: Serum alanine aminotransferase; AST: Serum aspartate aminotransferase; LKR: Sri Lankan rupees; RCTs: Randomised controlled trials; ICER: Incremental cost effectiveness ratio.
\end{abstract}

\section{Acknowledgements}

The authors are grateful to director and the consultant physicians of the NHSL for providing facility to carry out the study in the hospital. We are also thankful to medical, nursing and other staff of medical wards and staff of record room, accounts, salaries, and transport divisions of NHSL for providing assistance to do the data collection. We are also thankful to Professor Andrew Dawson and South Asian Clinical Toxicology Research Collaboration for funding.

\section{Author details}

'Department of Pharmacology, Faculty of Medicine, University of Colombo, Colombo, Sri Lanka. ${ }^{2}$ South Asian Clinical Toxicology Research Collaboration,
Kandy, Sri Lanka. ${ }^{3}$ Pharmacy Program, Department of Medical Education and Health Sciences, Faculty of Medical Sciences, University of Sri Jayewardenepura, Nugegoda, Sri Lanka. ${ }^{4}$ Faculty of Medicine, University of New South Wales, Sydney, Australia.

\section{Authors' contributions}

SMDKGS participated in the design and planning of the study, drafted the study proposal, collected patient information, conducted the economic evaluation and interpreted results, wrote the first draft and took part in revising the paper and finalizing the paper. SSR participated in the design and planning of the study, edited and revised the study proposal, edited the paper and took part in revising the paper and approved the final version. NB participated in the design and planning of the study, edited and revised the study proposal, edited the paper and took part in revising the paper and approved the final version. RF participated in the design and planning of the study, edited and revised the study proposal, edited the paper and took part in revising the paper and approved the final version. All authors read and approved the final manuscript.

Study design

Cost effectiveness analysis based on exploratory analysis of a systematic review

\section{Competing interests}

The authors declare that they have no competing interests.

Received: 25 February 2010 Accepted: 22 February 2012

Published: 22 February 2012

\section{References}

1. Sanaei-Zadeh $\mathrm{H}$, Tagghaddosinejad F, Jalali N, Kariman H: Adverse effects of intravenous N-acetylcysteine. Clin Drug Investig 2003, 23(2):129-133.

2. Buckley N, Eddleston M: Paracetamol (acetaminophen) poisoning. Clin Evid 2005, 14:1738-1744.

3. Senarathna SMDKG, Sri Ranganathan S, Dawson AH, Buckley N, Fernandopulle BMR: Management of acute paracetamol poisoning patients in a tertiary care hospital. Ceylon Med J 2008, 53(3):89-92.

4. Wickramasinghe K, Steele P, Dawson A, Dharmaratne D, Gunawardena A, Senarathna $L$, et al: Cost to government health-care services of treating acute self-poisonings in a rural district in Sri Lanka. Bull World Health Organ 2009, 87:180-185.

5. Senarathna SMDKG, Sri Ranganathan S, Fernandopulle BMR: Cost-outcome description of management of patients with acute paracetamol poisoning [Abstract]. Ceylon Med J 2008, 53(Suppl 1):53.

6. Brok J, Buckley N, Gluud C: Interventions for paracetamol (acetaminophen) overdoses. Cochrane Database Syst Rev 2002, 3, doi:10.1002/14651858.CD003328. Art No: CD003328.

7. Brok J, Buckley N, Gluud C: Interventions for paracetamol (acetaminophen) overdoses (Review). Cochrane Database Systemic Review 2006, 2, doi:10.1002/14651858.CD003328.pub2. Art. No.: CD003328.

8. Investing in Health: World Development Report Oxford: Oxford. University Press; 1993, 213-25, Ref Type: Report.

9. Prescott LF, Illingworth RN, Critchely JA, Stewart MJA, Proud foot AT: Intravenous Nacetylcysteine: the treatment of choice for paracetamol poisoning. Br Med J 1979, 2(6198):1097-1100.

10. Smilkstein MJ, Knapp Gl, Kulig KW, Rumack BH: Efficacy of oral Nacetylcysteine in the treatment of acetaminophen overdose. Analysis of a national multi centre study (1976-1985). N Engl J Med 1988, 319(24):1557-1562

11. Burkhart KK, Janco N, Kulig KW, Rumack BH: Cimetidine as adjunctive treatment for acetaminophen overdose. Hum Exp Toxicol 1995, 14(3):299-304.

12. Buckley NA, Whyte IM, O'Connell DL, Dawson AH: Oral or intravenous Nacetylcysteine: which is the treatment of choice for acetaminophen (paracetamol) poisoning. J Toxicol Clin Toxicol 1999, 37(6):759-767.

13. Parker $D$, White $P$, Paton $D$, Routledge A: Safety of late acetylcysteine treatment in paracetamol poisoning. Hum Exp Toxicol 1990, 9:25-27.

14. Smilkstein MJ, Bronskin AC, Linden C, Augenstein WL, Kulig KW, Rumack BH: Acetaminophen overdose: A 48-h intravenous $\mathrm{N}$-acetylcysteine treatment protocol. Ann Emerg Med 1991, 20:1058-1063.

15. Woo OF, Mueller PD, Olson KR, Anderson IB, Kim SY: Shorter duration of oral $\mathrm{N}$-acetylcysteine therapy for acute acetaminophen overdose. Ann Emerg Med 2000, 35(4):363-368. 
16. Ayonrinde OT, Phelps GJ, Hurley JC, Ayonrinde OA: Paracetamol overdose and hepatotoxicity at a regional Australian hospital: a 4 year experience. Intern Med J 2005, 35:655-660.

17. Kerr F, Dawson A, Whyte IM, Buckley N, Murray L, Graudins A, et al: The Australian clinical toxicology Investigators collaboration, Randomised Trails of Different loading infusion rates of Nacetylcysteine. Ann Emerg Med 2005, 45(4):402-408.

18. Crome P, Valem JA, Volans GN, Widdop B, Goulding R: Oral methionine in the treatment of severe paracetamol (Acetaminophen) overdose. Lancet 1976, 2(7990):829-830.

19. Hamlyn AN, Lesna M, Record CO, Smith PA, Path FRC, Watson AJ: Methionine and cysteamine in paracetamol overdose, prospective controlled trial of early therapy. J Int Med Res 1981, 9:226-231.

20. Prescott LF, Park J, Sutherland GR, Smith IJ: Cysteamine, methionine, and penicillamine in the treatment of paracetamol poisoning. Lancet 1976, 2:109-113.

21. Vale JA, Meredith TJ, Goulding R: Treatment of acetaminophen poisoning. The use of oral methionine. Arch Intern Med 1981, 141(3):394-396.

22. National Institute for Clinical Excellence (NICE): Guide to the methods of technology appraisal London London: NICE; 2004.

23. Census and statistics Sri Lanka: Household income and expenditure survey 2006/07 summary findings. [On line]. Department of Census and statistics Sri Lanka 2007, Available at: http://www.statistics.gov.lk/HIES/ HIES2006_07Website/Publications/SummaryFfindingsHIES2006_07.pdf [Accessed on 1st April 2008].

24. Cook DJ, Guyatt GH, Laupacis A, Sackett DL: Rules of evidence and clinical recommendations on the use of antithrombitic agents. Chest 1992, 102:305s-311s.

\section{Pre-publication history}

The pre-publication history for this paper can be accessed here:

http://www.biomedcentral.com/1472-6904/12/6/prepub

doi:10.1186/1472-6904-12-6

Cite this article as: Senarathna et al: A cost effectiveness analysis of the preferred antidotes for acute paracetamol poisoning patients in Sri Lanka. BMC Clinical Pharmacology 2012 12:6.

\section{Submit your next manuscript to BioMed Central and take full advantage of:}

- Convenient online submission

- Thorough peer review

- No space constraints or color figure charges

- Immediate publication on acceptance

- Inclusion in PubMed, CAS, Scopus and Google Scholar

- Research which is freely available for redistribution

Submit your manuscript at www.biomedcentral.com/submit
Biomed Central 